\title{
REVISED Improved deconvolution of very weak confocal signals
}

\section{[version 2; peer review: 2 approved]}

\author{
Kasey J. Day¹, Patrick J. La Rivière², Talon Chandler², Vytas P. Bindokas³, \\ Nicola J. Ferrier, Benjamin S. Glick (iD)1 \\ ${ }^{1}$ Department of Molecular Genetics and Cell Biology, University of Chicago, Chicago, IL, 60637, USA \\ 2Department of Radiology, University of Chicago, Chicago, IL, 5841, USA \\ 3Integrated Light Microscopy Core Facility, University of Chicago, Chicago, IL, 60637, USA \\ ${ }^{4}$ Computation Institute, University of Chicago Mathematics and Computer Science, Argonne National Laboratory, Argonne, IL, \\ 60439, USA
}

\author{
V2 First published: 06 Jun $2017,6: 787$ \\ https://doi.org/10.12688/f1000research.11773.1 \\ Latest published: 07 Aug 2017, 6:787 \\ https://doi.org/10.12688/f1000research.11773.2
}

\section{Abstract}

Deconvolution is typically used to sharpen fluorescence images, but when the signal-to-noise ratio is low, the primary benefit is reduced noise and a smoother appearance of the fluorescent structures. 3D time-lapse (4D) confocal image sets can be improved by deconvolution. However, when the confocal signals are very weak, the popular Huygens deconvolution software erases fluorescent structures that are clearly visible in the raw data. We find that this problem can be avoided by prefiltering the optical sections with a Gaussian blur. Analysis of real and simulated data indicates that the Gaussian blur prefilter preserves meaningful signals while enabling removal of background noise. This approach is very simple, and it allows Huygens to be used with 4D imaging conditions that minimize photodamage.

\section{Keywords}

deconvolution, Gaussian blur, fluorescence microscopy, confocal

microscopy, 4D microscopy, signal-to-noise, Huygens

\section{Open Peer Review \\ Approval Status \\ 1 2 \\ version 2 \\ (revision) \\ 07 Aug 2017 \\ version 1 \\ 06 Jun 2017

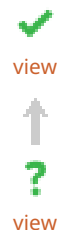

1. Akihiko Nakano ID, RIKEN Center for Advanced Photonics, Wako, Japan

Daisuke Miyashiro, RIKEN Center for Advanced Photonics, Wako, Japan

2. Vladimir Denic, Harvard University,

Cambridge, USA

Nicholas Weir, Harvard University,

Cambridge, USA

Any reports and responses or comments on the article can be found at the end of the article. 
Corresponding author: Benjamin S. Glick (bsglick@uchicago.edu)

Author roles: Day KJ: Conceptualization, Formal Analysis, Funding Acquisition, Investigation, Methodology, Project Administration, Resources, Supervision, Writing - Original Draft Preparation, Writing - Review \& Editing; La Rivière PJ: Conceptualization, Formal Analysis, Investigation, Methodology, Validation, Visualization, Writing - Review \& Editing; Chandler T: Conceptualization, Formal Analysis, Investigation, Methodology, Validation, Visualization, Writing - Review \& Editing; Bindokas VP: Conceptualization, Formal Analysis, Investigation, Methodology, Software, Validation; Ferrier NJ: Formal Analysis, Investigation, Methodology, Validation, Visualization, Writing - Review \& Editing; Glick BS: Formal Analysis, Investigation, Methodology, Validation, Writing - Review \& Editing

Competing interests: No competing interests were disclosed.

Grant information: Funding was provided through the Biological Systems Science Division, Office of Biological and Environmental Research, Office of Science, U.S. Dept. of Energy, under Contract DE-AC02-06CH11357.

The funders had no role in study design, data collection and analysis, decision to publish, or preparation of the manuscript.

Copyright: ( 2017 Day KJ et al. This is an open access article distributed under the terms of the Creative Commons Attribution License, which permits unrestricted use, distribution, and reproduction in any medium, provided the original work is properly cited. Data associated with the article are available under the terms of the Creative Commons Zero "No rights reserved" data waiver (CCO 1.0 Public domain dedication).

How to cite this article: Day KJ, La Rivière PJ, Chandler T et al. Improved deconvolution of very weak confocal signals [version 2; peer review: 2 approved] F1000Research 2017, 6:787 https://doi.org/10.12688/f1000research.11773.2

First published: 06 Jun 2017, 6:787 https://doi.org/10.12688/f1000research.11773.1 


\section{REVISED Amendments from Version 1}

This revision has been prepared under somewhat unusual circumstances. After seeing the original version of the paper, scientists at SVI analyzed our image data and determined that deconvolution of very weak confocal signals could be improved by refining the background estimation procedure in Huygens. We find that this change completely solves the problems reported here for both real and simulated confocal data.

This outcome is ideal because it ensures that we and others will obtain excellent results with Huygens even under suboptimal imaging conditions. With newer versions of Huygens, the Gaussian blur prefilter is no longer needed. Given these developments, extensive revision of the paper would be hard to justify. We therefore limited the changes to a few textual modifications that improve clarity and accuracy based on feedback from reviewers, readers, and scientists at SVI. An "Update" section at the end summarizes the recent developments.

For researchers running older versions of Huygens, the Gaussian blur prefilter is still an effective method for processing very weak confocal data. In addition, this paper and the associated raw data may prove to be useful for "stress testing" other deconvolution algorithms.

See referee reports

\section{Introduction}

Deconvolution is an established method for sharpening fluorescence images and removing background noise (Biggs, 2010; Sage et al., 2017). The usual input to a deconvolution algorithm is a Z-stack of optical sections generated by widefield or confocal microscopy. Because the benefits of deconvolution are fully realized when the signals are strong, the creators of deconvolution software recommend capturing a large number of photons while keeping the pixel sizes and Z-step intervals relatively small.

Those conditions are hard to meet with live cell imaging if Z-stacks are being collected at regular intervals to create a 3D time-lapse (4D) data set (De Mey et al., 2008). Intracellular structures are dynamic, so the images need to be taken rapidly. Moreover, the number of captured photons is severely constrained by the need to avoid photodamage to the cells and fluorophores (Carlton et al., 2010; Pawley, 2006). Such issues are prominent in our 4D confocal microscopy studies of secretory compartments in yeast cells (Bevis et al., 2002; Losev et al., 2006; Papanikou et al., 2015). We maximize the scan speed, minimize the intensities of the excitation lasers, and set the pixel sizes and Z-step intervals at the traditionally defined Nyquist limit to achieve a tolerable light exposure while ensuring accurate representation of the imaged structures (Day et al., 2016; Pawley, 2006). The resulting data sets typically comprise thousands of optical sections and have a low signal-to-noise ratio (SNR).

Even though the sampling characteristics of our 4D data are not ideal for deconvolution, the Huygens deconvolution software from Scientific Volume Imaging (SVI) can facilitate the analysis. A number of other freeware and commercial software packages are also available for deconvolution (Biggs, 2010; Sage et al., 2017), but in our experience, those programs are unsuitable for processing of multi-channel 4D confocal data due to some combination of cumbersome user interfaces, lack of compatibility with relevant file formats, and inadequate noise removal. Huygens is unique in that it readily deconvolves our data sets (Day et al., 2016; Papanikou et al., 2015). This software is widely used in the cell biology research community. Importantly, in addition to removing noise, Huygens smooths the uneven shapes and intensities obtained with low-SNR data to generate images that are easy to view and quantify.

Huygens works well for certain low-SNR fluorescence images, but when the fluorescence signals are very weak, Huygens may perform poorly (Arigovindan et al., 2013). We encountered this problem when imaging low-abundance proteins associated with yeast organelles. In such experiments, a pixel in the signalcontaining portion of a confocal section may capture as few as 1-2 photons. To enable deconvolution of images with a very low SNR, Agard and colleagues developed deconvolution software called ER-Decon, which employs a novel regularization method tailored to fluorescence data (Arigovindan et al., 2013). However, ER-Decon has incompletely defined parameters, and it proved to be challenging to use. We therefore sought a method for processing very weak fluorescence signals with Huygens.

\section{Methods}

Confocal microscopy and image processing

4D imaging of live yeast cells expressing Vps8-GFP and Sec7mCherry was performed as previously described (Day et al., 2016) with a Leica SP5 confocal microscope. Briefly, images were collected at the maximum scan speed with a $63 \mathrm{x} 1.4$ NA objective using a voxel size of $80 \times 80 \times 250 \mathrm{~nm}$, a pinhole setting of 1.2 Airy units, and HyD hybrid detectors in photon counting mode. Z-stacks of 28 optical sections were captured at $2 \mathrm{~s}$ intervals with the line accumulation (summing) set to either $8 \mathrm{x}$ or $1 \mathrm{x}$. Image manipulations other than deconvolution, including $2 \mathrm{D}$ and $3 \mathrm{D}$ Gaussian blurs, employed 64-bit ImageJ 1.51i (http://rsbweb.nih. gov/ij/) (RRID: SCR_003070). This software has a sophisticated Gaussian blur algorithm that chooses a suitable kernel based on the user-specified radius (sigma) value. Multi-channel 8-bit confocal 3D time series data were converted to TIFF format, and the TIFF images were converted to 16-bit format, multiplied by 256, and Gaussian blurred where indicated. After deconvolution, the image stacks were average projected and then scaled to provide a quantitatively accurate view of the fluorescent structures (Hammond \& Glick, 2000), and the series of projections was exported to AVI movie format. An online tool was used to convert the movies to MP4 format (http://video.online-convert.com/ convert-to-mp4).

For labeling the yeast nuclear envelope and peripheral ER membranes, gene replacement was used to tag Hmg1 with GFP. The accompanying pHMG1-GFP.dna SnapGene file (Supplementary File 1) shows the plasmid used for the strain construction. That file can be opened with SnapGene Viewer (http://www.snapgene. com/products/snapgene_viewer/). The construction steps can be visualized using History view, and instructions for tagging Hmg1 
by gene replacement can be found in the Description Panel. Confocal imaging of yeast cells expressing Hmg1-GFP was performed with a Leica SP8 confocal microscope using the same parameters as for 4D imaging, except that 31 optical sections were captured. A series of 14 optical sections (numbers 13-26) representing approximately $3 \mu \mathrm{m}$ from the central portions of the cells were processed and average projected as described above.

\section{Simulation of weakly fluorescent objects}

A simulated point-like fluorescent object was generated in a voxel array of XYZ dimensions 200x200x40 with a voxel size of $80 \times 80 \times 250 \mathrm{~nm}$. The fluorescent object was centered along the Z-axis, and was duplicated at an XY spacing of 20 pixels to create an $8 \times 8$ array.

The effective confocal point spread function (PSF) was generated by multiplying simulated excitation and emission PSFs, which were produced by the ImageJ plugin PSF Generator (http://bigwww.epfl.ch/algorithms/psfgenerator/) using the Born \& Wolf 3D optical model. This model is appropriate for an object located next to the coverslip. The plugin was used with the following parameters: refractive index $=1.5$, numerical aperture $=$ 1.4 , voxel size $=80 \times 80 \times 250 \mathrm{~nm}$, excitation wavelength $=488 \mathrm{~nm}$, emission wavelength $=510 \mathrm{~nm}$.

The effective confocal PSF was convolved with the simulated objects using fast Fourier transform-based 3D convolution, and the image values were normalized so that the maximum pixel value corresponded to an average of 1 detected photon. The resulting image stack represented the average detected image, which comprised a total of 17.7 photons per object. Where indicated, random background noise was included by adding a value of 0.01 photons to every voxel in the average detected image. This information was used as input to a Poisson random number generator, yielding a simulated image stack in which each voxel value was drawn from a Poisson distribution whose mean was equal to the corresponding voxel value in the average detected image.

The output was saved in 8-bit TIFF format, and was scaled so that a pixel value of 255 corresponded to 4 photons. Further processing was carried out as for the live cell confocal data, except that Gaussian blurring and/or deconvolution were performed with 8-bit format. The images were then converted to 16-bit format and multiplied by 256 followed by average projection.

To quantify the signal intensity for an object after average projection, ImageJ was used to create a selection of $20 \times 20$ pixels centered on the object, and the integrated density was measured. For the deconvolved image, the numbers were multiplied by a correction factor to compensate for scaling of the image by Huygens.

\section{Deconvolution}

Deconvolution with Huygens Essential 15.10 software (https:// svi.n1/HomePage) (RRID: SCR_014237) was performed on an iMac using up to 40 iterations of the Classic Maximum Likelihood Estimation algorithm with a theoretical PSF. Background correction was automatic, except in the case of the simulated confocal Z-stacks with added background noise, for which the background setting was manually adjusted to 0.8 . The SNR setting, adjusted empirically to give satisfactory results, was as follows: 4 for the live cell 4D confocal data; 7 for the confocal images of cells with a labeled nuclear envelope; 7 for the simulated confocal Z-stacks with no added background noise; 1 for the simulated confocal Z-stack with added background noise; or 10 for the widefield data. The other parameters used by the Huygens algorithm were configured for either confocal microscopy of live yeast cells (Day et al., 2016), confocal microscopy of simulated fluorescent objects under the conditions specified during the simulation, or widefield microscopy under the conditions reported for the ER-Decon software (Arigovindan et al., 2013).

The ER-Decon software and associated image data were obtained from the University of California, San Francisco (http:// msg.ucsf.edu/IVE/Download/). Images of fluorescent yeast Zip1 filaments were obtained as part of the ER-Decon package, and were converted to TIFF format using the Bio-Formats Importer plugin for ImageJ (http://www.openmicroscopy.org/site/support/ bio-formats5.1/).

\section{Results and discussion}

We generated two small 4D data sets to illustrate confocal imaging of organelles in live Saccharomyces cerevisiae cells. The parameters were adjusted to capture either weak signals using a line accumulation of $8 x$, where each line in the image was scanned eight times and the results were summed (Video S1), or very weak signals using a line accumulation of $1 \mathrm{x}$, where each line in the image was scanned only once (Video S2). Projections of representative Z-stacks from the two movies are shown in Figure 1. The organelles were dynamic, so the labeling patterns in the two movies are not identical, but the movies were brief and were captured sequentially, so the labeling patterns are similar enough to allow for comparison. With $8 x$ line accumulation, the raw projections are noisy and display fluorescent structures with uneven shapes and intensities (Video S1 and Figure 1A). Deconvolution with Huygens efficiently removed the background noise and smoothed the structures. With $1 \mathrm{x}$ line accumulation, the data quality is even lower, but fluorescent structures can still be discerned in the raw projections (Video S2 and Figure 1B). In this case, deconvolution with Huygens erased almost all of the fluorescent structures. The processing employed standard settings in the Huygens software, including deconvolution with the Classic Maximum Likelihood Estimation algorithm. Although a larger percentage of the fluorescent structures in very weak data sets could be preserved by greatly reducing the number of deconvolution iterations or by using different SNR or background settings, the preserved structures often had distorted shapes (not shown). Similar loss or poor preservation of very weak fluorescent structures was seen with the Good's roughness Maximum Likelihood Estimation algorithm, which is recommended for use with noisy confocal data (not shown). Based on these observations, we have continued to use standard settings in Huygens. Our data sets often lie between the two extremes depicted in Figure 1, and when movies are generated after deconvolution, the fluorescent structures blink because a given structure is erased in some movie frames but not in others (see Video S2). Such movies cannot be productively analyzed. 
A

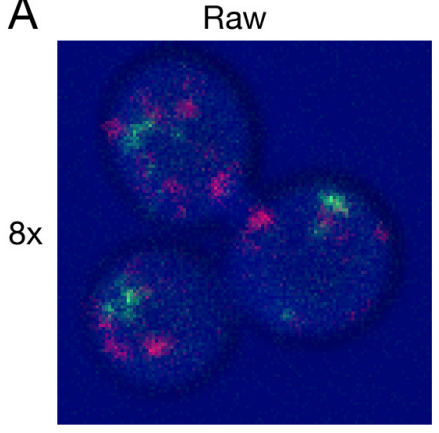

B

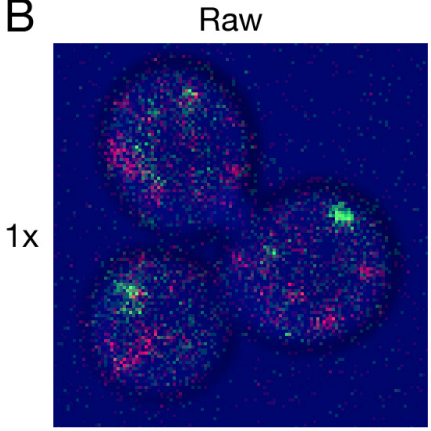

Deconvolved

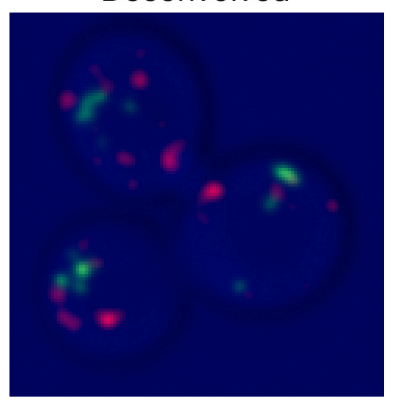

Deconvolved

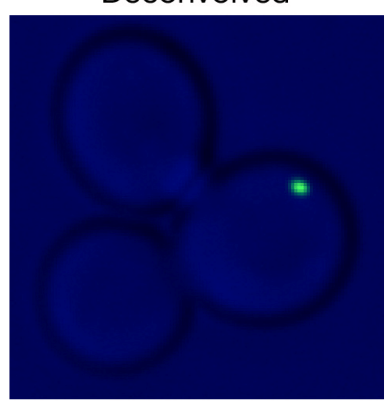

Blurred, Deconvolved

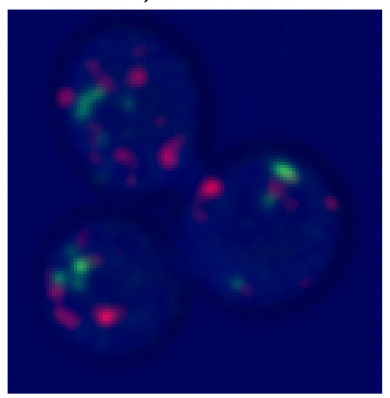

Blurred, Deconvolved

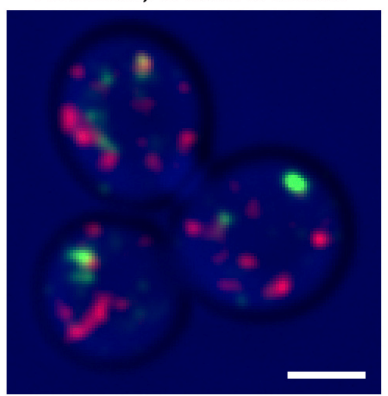

Figure 1. Improved deconvolution of 4D live cell data with a Gaussian blur prefilter. Gene replacement in Saccharomyces cerevisiae was used to label late Golgi cisternae with Sec7-mCherry (red) and prevacuolar endosomes with Vps8-GFP (green) (Papanikou et al., 2015). Cells were imaged by $4 \mathrm{D}$ confocal microscopy. In consecutive movies, line accumulation was set to (A) $8 x$ or (B) $1 x$. The data were average projected either with no processing, or after deconvolution with Huygens, or after prefiltering with a 2D Gaussian blur using a radius of 0.75 pixels followed by deconvolution. Fluorescence data are superimposed on differential interference contrast images of the cells (blue). Shown are representative frames from Video $1(8 \mathrm{x})$ and Video $2(1 \mathrm{x})$. The fluorescence patterns in $(\mathbf{A})$ and $(\mathbf{B})$ are similar, but not identical because the labeled structures changed during the interval between the two movies. Scale bar, $2 \mu \mathrm{m}$.

Dataset 1.TIFF files for the experimental and simulated image data are provided in the compressed folder Original Image Files.zip

http://dx.doi.org/10.5256/f1000research.11773.d163336

The following files are included: 4D_movie_1x.tif and 4D_ movie_8x.tif are the 4D confocal data sets used for Figure 1 and Supplementary Figure 1, and for Video S1 and Video S2; Hmg1_1x.tif and Hmg1_8x.tif are the confocal image stacks used for Figure 2; simulation_80x80x250.tif is the simulated confocal image stack used for Figure 3A and Supplementary Figure 2; simulation $80 \times 80 \times 250$ plus noise.tif is the simulated confocal

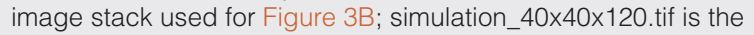
simulated confocal image stack used for Supplementary Figure 3; and Zip1_0.25\%.tif and Zip1_100\%.tif are the widefield image stacks used for Figure 4.

In the course of testing several types and combinations of image filters (Day et al., 2016), we discovered that for very weak confocal signals, the key step was to prefilter the optical sections in ImageJ with a Gaussian blur. That prefilter dramatically improved the results obtained after deconvolution (Video S2 and Figure 1B). Fluorescent structures were no longer erased, and instead were preserved and smoothed while the background noise was largely eliminated. Most of the structures visualized by this method were biologically relevant because they persisted between movie frames (Video S2). Essentially identical results were obtained with 2D and 3D Gaussian blurs (not shown), so we use a 2D Gaussian blur because the processing is faster. This prefiltering step enables us to generate useful 4D movies from data sets that contain very weak confocal signals.

Application of the Gaussian blur prefilter requires the data to be in a suitable format. Our images are collected with a high-sensitivity detector in photon counting mode, and the pixel values are in 8-bit format. For very weak signals, typical pixel values are 0 , 1 , or 2 because a pixel rarely captures more than 2 photons. To obtain a meaningful blur, the numbers are scaled up to allow for intermediate integer values. We convert the images to 16-bit format and multiply by 256 , resulting in typical pixel values of 0,256 , and 512. A Gaussian blur then generates a range of intermediate values, effectively spreading the individual photon signals over multiple pixels.

An important question is how to determine whether a confocal data set is suitable for processing with a Gaussian blur prefilter. Ideally, this prefilter would be used routinely, because even if the average signal intensity is strong, some structures may have very 
weak signals. The concern with routine application of a Gaussian blur prefilter is that blurring might be propagated to the deconvolved images. Indeed, when the Gaussian blur prefilter was applied to signals strong enough to be preserved during normal deconvolution, we saw some blurring of the fluorescent structures (Video S1 and Figure 1A). However, this effect was minor with suitable parameters for the prefilter (see below). Our results indicate that a Gaussian blur prefilter can be used to image structures with a range of signal intensities, resulting in preservation of very weak signals without significant degradation of stronger signals.

Because the labeled structures in our 4D data sets were punctate, we tested whether a Gaussian blur prefilter would also improve deconvolution of other shapes. For this purpose, GFP was fused to a yeast endoplasmic reticulum (ER) protein that localizes mainly to the nuclear envelope (Koning et al., 1996). A single confocal Z-stack was captured at a low excitation laser setting. As shown in Figure 2A, which employed 8x line accumulation, the labeled protein appeared as prominent nuclear envelope rings with weaker labeling of peripheral ER membranes. Deconvolution of the raw data preserved the nuclear envelope rings. When a Gaussian blur was applied before deconvolution, additional signals outside the nuclear envelope were preserved. Figure 2B shows a parallel analysis with $1 \mathrm{x}$ line accumulation. In this case, the fluorescence signals were completely erased by deconvolution of the raw data, but application of a Gaussian blur before deconvolution preserved the nuclear envelope rings. We conclude that for various types of fluorescence patterns, a Gaussian blur prefilter preserves very weak confocal signals during deconvolution with Huygens.

To explore the Gaussian blur effect systematically, and to confirm that it was not limited to the particular configuration of our confocal microscopy setup, we used simulated data. 3D confocal imaging was simulated for an array of 64 faintly fluorescent point-like objects, each of which was represented by about 10-25 photons spread over multiple optical sections. Figure $3 \mathrm{~A}$ shows projections of this simulated Z-stack before and after processing. After deconvolution with Huygens, only 7 objects were preserved, but after a Gaussian blur prefilter followed by deconvolution, all 64 objects were preserved. The total signal intensities for the objects were largely unchanged after either a Gaussian blur alone or a Gaussian blur followed by deconvolution (Supplementary Figure 1). A setting of 0.75 pixels for the radius (sigma) parameter of the prefilter preserved signals while causing very little blur in the final images, and similar results were obtained with a radius of 1.00 pixels (Supplementary Figure 2). We find empirically that radius values of $0.75-1.00$ pixels work well for both real and simulated fluorescence data obtained under a variety of imaging conditions. When the simulation was repeated with added background noise, a Gaussian blur prefilter followed by deconvolution removed most of the noise while preserving all of the objects (Figure 3B). In this case, deconvolution in the absence of a prefilter completely erased the objects. The voxels in those simulations were $80 \times 80 \times 250 \mathrm{~nm}$ to mimic traditional Nyquist imaging with our confocal system (Pawley, 2006), but similar results were obtained with voxels of 40x40x120 nm (Supplementary Figure 3) chosen to meet the more stringent Nyquist criteria recommended by SVI. Thus, a Gaussian blur prefilter preserves weak confocal signals during deconvolution under multiple real and simulated conditions.

The paper describing the ER-Decon software showed that Huygens could give unsatisfactory results with low-SNR widefield images (Arigovindan et al., 2013). We processed low-SNR widefield microscopy data from that study with a Gaussian blur prefilter before deconvolution. The improvement was only moderate because Huygens did not erase the structures, but when the signal was weak, the prefilter did increase contrast between labeled structures and the background (Figure 4, right panels), yielding results similar to those obtained with ER-Decon (compare to Figure 4 in
A

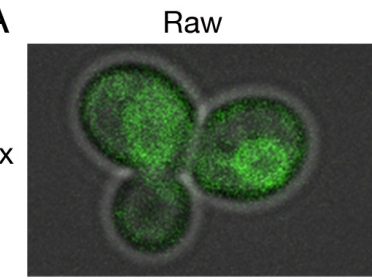

B

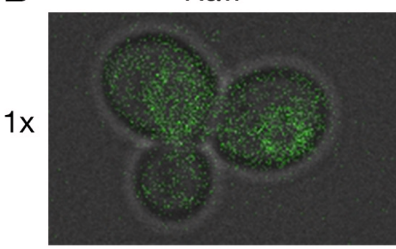

Deconvolved

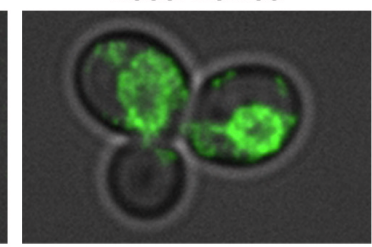

Deconvolved

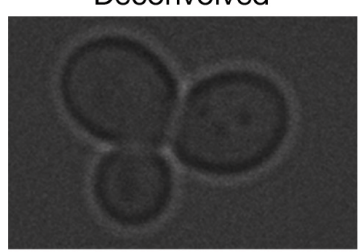

Blurred, Deconvolved

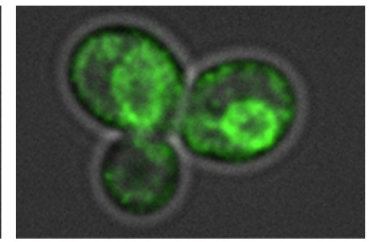

Blurred, Deconvolved

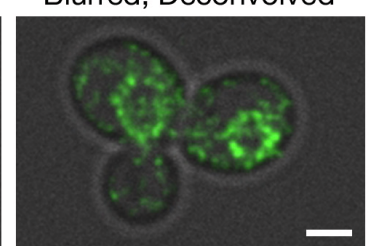

Figure 2. Improved deconvolution of non-punctate fluorescence signals with a Gaussian blur prefilter. Gene replacement in Saccharomyces cerevisiae was used to label ER membranes with Hmg1-GFP (Koning et al., 1996). A confocal Z-stack was captured with line accumulation set to $(\mathbf{A}) 8 x$ or $(\mathbf{B})$ 1x. The data were average projected either with no processing, or after deconvolution with Huygens, or after prefiltering with a 2D Gaussian blur using a radius of 0.75 pixels followed by deconvolution. Fluorescence data are superimposed on differential interference contrast images of the cells (gray). Scale bar, $2 \mu \mathrm{m}$. 
A

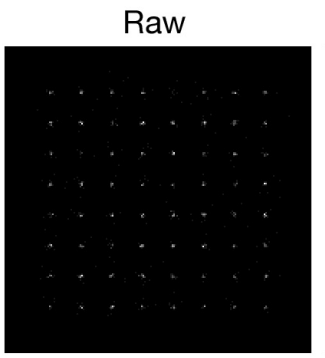

B

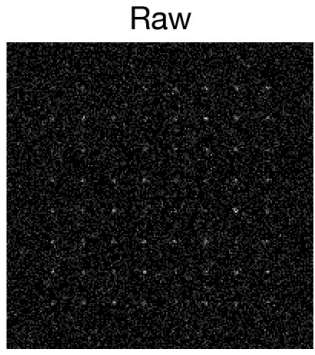

Deconvolved

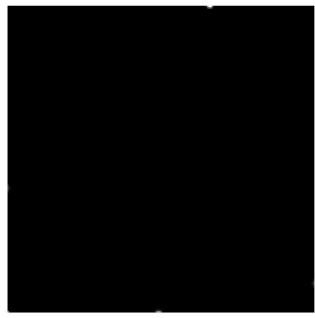

Deconvolved
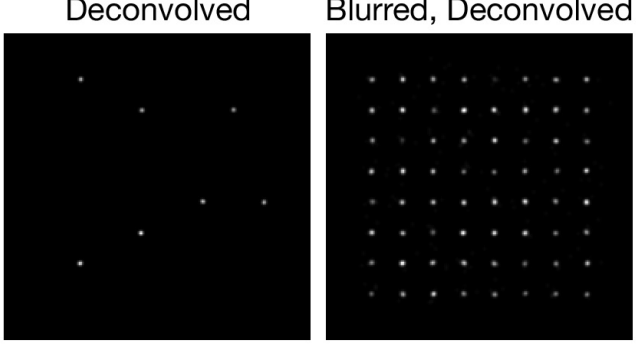

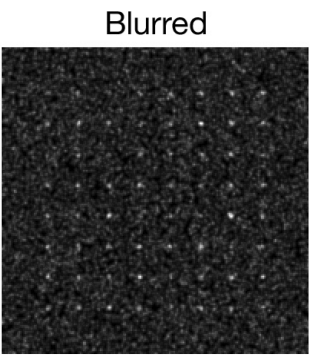

Blurred, Deconvolved

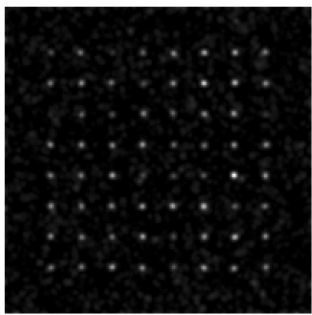

Figure 3. Improved deconvolution of simulated data with a Gaussian blur prefilter. Simulated confocal Z-stacks of fluorescent point sources were created as described in Methods, either $(\mathbf{A})$ without background noise or $(\mathbf{B})$ with background noise. The data were processed and average projected as in Figure 1.

Arigovindan et al., 2013). The combined observations demonstrate that a Gaussian blur prefilter consistently improves deconvolution of low-SNR fluorescence data.

The reason for this beneficial effect of the prefilter is not fully understood. Gaussian blurs suppress high-frequency noise. That approach reduces pixel-to-pixel intensity variations, and it can facilitate analysis methods such as edge detection and particle tracking (Cheezum et al., 2001; Russ \& Neal, 2015). A different mechanism presumably underlies the ability of a Gaussian blur to prevent loss of very weak signals during deconvolution. Huygens apparently "expects" a gradually varying distribution of the signal intensities within a set of nearby voxels, and the Gaussian blur prefilter converts the data to a form suitable for the Huygens algorithm.

Is a Gaussian blur prefilter before deconvolution an acceptable procedure? Processing of images before deconvolution is not generally recommended, but a Gaussian blur is relatively safe. This filter causes a simple and well behaved transformation of the data, and it preserves the total intensity of a fluorescent structure (Burger \& Burge, 2008). Gaussian blurs have previously been employed during deconvolution to suppress noise buildup (Agard et al., 1989). A Gaussian blur prefilter was actually proposed by the founder of SVI as a method that can reduce noise sensitivity during deconvolution of confocal data (van Kempen et al., 1997). Therefore, it seems reasonable to apply this prefilter to very weak confocal signals for the novel purpose of avoiding complete erasure of biologically meaningful structures. When the signals are stronger, the Gaussian blur prefilter has a barely detectable effect on the final image, so there seems to be little risk in applying this prefilter routinely.

It could be argued that the Gaussian blur prefilter merely sidesteps a software flaw, in which case a better option would be to fix the Huygens algorithm. However, Huygens is optimized for processing images that exceed a minimum signal strength, and our confocal data sometimes fall below this threshold. Other deconvolution algorithms may perform differently. The available evidence specifically shows that the Gaussian blur prefilter is useful with Huygens. This straightforward method allows us to take advantage of the flexibility, noise removal capability, and smoothing properties of the Huygens software to process very weak fluorescence signals. 

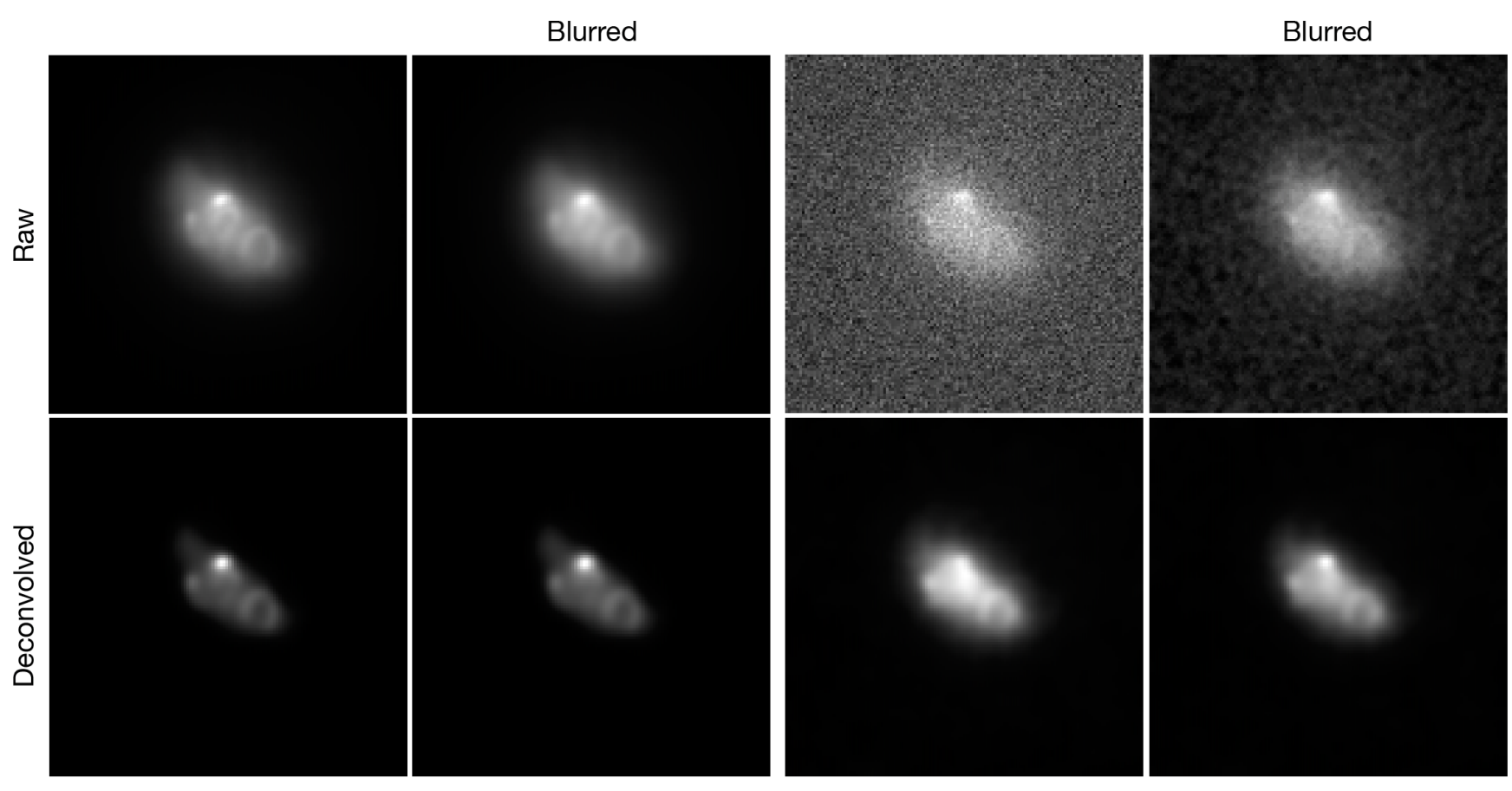

Figure 4. Effect of a Gaussian blur prefilter on deconvolution of widefield fluorescence data. These images of fluorescent yeast Zip1 filaments correspond to Figure 4 of Arigovindan et al. (2013). The two exposure levels represent strong (100\%) or weak (0.25\%) signals, respectively. Where indicated, the data were subjected either to a Gaussian blur with a radius of 1.00 pixel, or to deconvolution with Huygens, or to a Gaussian blur prefilter followed by deconvolution. The theoretical point spread function was based on imaging parameters supplied with ER-Decon.

\section{Update}

After seeing the initial version of this manuscript, scientists at SVI analyzed the performance of Huygens with our data sets, and refined their background estimation procedure to improve the deconvolution of images with very weak signals. We find that this change produces excellent results with both real and simulated confocal data obtained under our imaging conditions. The deconvolved images reliably preserve the signals while avoiding the slight loss of sharpness that was observed with a Gaussian blur prefilter. We are grateful to SVI for their responsiveness and constructive feedback.

The improved algorithm is available in Huygens versions 17.04 and later. For investigators running earlier versions of Huygens, the Gaussian blur prefilter remains an effective option for preserving very weak confocal signals during deconvolution. Moreover, the Gaussian blur prefilter may prove to be beneficial for other types of images that are sampled in a manner not well suited to direct processing with Huygens.

\section{Data availability}

Dataset 1: TIFF files for the experimental and simulated image data are provided in the compressed folder Original Image Files.zip. The following files are included: 4D_movie_ 1x.tif and 4D_movie_8x.tif are the 4D confocal data sets used for Figure 1 and Supplementary Figure 1, and for Video S1 and Video S2; Hmg1_1x.tif and Hmg1_8x.tif are the confocal image stacks used for Figure 2; simulation_80x80x250.tif is the simulated confocal image stack used for Figure 3A and Supplementary Figure 2; simulation_80x80x250_plus_noise.tif is the simulated confocal image stack used for Figure 3B; simulation_40x40x120. tif is the simulated confocal image stack used for Supplementary Figure 3; and Zip1_0.25\%.tif and Zip1_100\%.tif are the widefield image stacks used for Figure 4. doi, 10.5256/f1000research.11773. d163336 (Day et al., 2017).

\section{Competing interests}

No competing interests were disclosed.

\section{Grant information}

Funding was provided through the Biological Systems Science Division, Office of Biological and Environmental Research, Office of Science, U.S. Dept. of Energy, under Contract DEAC02-06CH11357.

The funders had no role in study design, data collection and analysis, decision to publish, or preparation of the manuscript.

\section{Acknowledgments}

Thanks for helpful discussion to the deconvolution experts at SVI and to Marc Bruce of Microvolution. 


\section{Supplementary material Supplementary File 1. SnapGene file for the Hmg1-GFP construct.}

Click here to access the data.

Supplementary Figure 1. Preservation of signal intensities after image processing. For the simulated array shown in Figure $3 \mathrm{~A}$, the intensities after average projection for the eight objects in the top row were measured using either the raw data, or the data after a Gaussian blur with a radius of 0.75 pixels, or the data after a Gaussian blur followed by deconvolution with Huygens. Intensity values are in arbitrary units.

Click here to access the data.

Supplementary Figure 2. Comparison of different radius values for the Gaussian blur prefilter. The simulated confocal Z-stack in Figure 3A was subjected to a Gaussian blur prefilter using the indicated radius (sigma) values in pixels, then deconvolved with Huygens and average projected. A radius of 0.50 was not completely effective at preserving the objects, and a radius of 1.00 caused slight blurring in the final image, indicating that a radius of 0.75 was a good compromise.

Click here to access the data.

Supplementary Figure 3. Effect of a Gaussian blur prefilter with a smaller voxel size. A simulated confocal Z-stack was generated and processed as in Figure 3A, except that the voxel size was 40x40x120 nm.

Click here to access the data.

Video S1. Movie generated with weak signals from labeled yeast organelles. Confocal Z-stacks were collected at $2 \mathrm{~s}$ intervals with a line accumulation setting of $8 \mathrm{x}$. The final frame of this movie corresponds to Figure 1A.

Click here to access the data.

Video S2. Movie generated with very weak signals from labeled yeast organelles. Confocal Z-stacks were collected at $2 \mathrm{~s}$ intervals with a line accumulation setting of $1 \mathrm{x}$. The final frame of this movie corresponds to Figure 1B.

Click here to access the data.

Agard DA, Hiraoka $Y$, Shaw $P$, et al: Fluorescence microscopy in three dimensions. In Fluorescence Microscopy of Living Cells in Culture, Part B. Methods Cell Biol. Taylor DL and Wang Y, editors. Academic Press, San Diego. 1989; 30: 353-377. PubMed Abstract | Publisher Full Text

Arigovindan M, Fung JC, Elnatan D, et al:: High-resolution restoration of 3D structures from widefield images with extreme low signal-to-noise-ratio. Proc Natl Acad Sci U S A. 2013; 110(43): 17344-17349. PubMed Abstract | Publisher Full Text | Free Full Text

Bevis BJ, Hammond AT, Reinke CA, et al:: De novo formation of transitional ER sites and Golgi structures in Pichia pastoris. Nat Cell Biol. 2002; 4(10): 750-756. PubMed Abstract | Publisher Full Text

Biggs DS: 3D deconvolution microscopy. Curr Protoc Cytom. 2010; Chapter 12 : Unit12.19.1-20.

PubMed Abstract | Publisher Full Text

Burger W, Burge MJ: Digital Image Processing: An Algorithmic Introduction using Java. Springer, New York NY. 2008.

Publisher Full Text

Carlton PM, Boulanger J, Kervrann C, et al.: Fast live simultaneous multiwavelength four-dimensional optical microscopy. Proc Natl Acad Sci U S A. 2010; 107(37): 16016-16022.

PubMed Abstract | Publisher Full Text | Free Full Text

Cheezum MK, Walker WF, Guilford WH: Quantitative comparison of algorithms for tracking single fluorescent particles. Biophys J. 2001; 81(4): 2378-2388. PubMed Abstract | Publisher Full Text | Free Full Text

Day KJ, Papanikou E, Glick BS: 4D Confocal Imaging of Yeast Organelles. Methods Mol Biol. 2016; 1496: 1-11.

PubMed Abstract | Publisher Full Text | Free Full Text

Day KJ, La Rivière PJ, Chandler T, et al.: Dataset 1 in: Improved deconvolution

of very weak confocal signals. F1000Research. 2017.

Data Source
De Mey JR, Kessler P, Dompierre J, et al: Fast 4D Microscopy. Methods Cell Biol. 2008; 85: 83-112.

PubMed Abstract | Publisher Full Text

Hammond AT, Glick BS: Raising the speed limits for 4D fluorescence microscopy. Traffic 2000: 1(12): 935-940.

PubMed Abstract | Publisher Full Text

Koning AJ, Roberts CJ, Wright RL: Different subcellular localization of Saccharomyces cerevisiae HMG-CoA reductase isozymes at elevated levels corresponds to distinct endoplasmic reticulum membrane proliferations. $\mathrm{MO}$ Biol Cell. 1996; 7(5): 769-789.

PubMled Abstract | Publisher Full Text | Free Full Text

Losev $\mathrm{E}$, Reinke $\mathrm{CA}$, Jellen J, et al:: Golgi maturation visualized in living yeast. Nature. 2006; 441(7096): 1002-1006.

PubMed Abstract | Publisher Full Text

Papanikou E, Day KJ, Austin J, et al:: COPI selectively drives maturation of the early Golgi. eLife. 2015; 4: pii: e13232.

PubMed Abstract | Publisher Full Text | Free Full Text

Pawley JB: Handbook of Biological Confocal Microscopy, Second Edition. Springer. 2006; 985.

Publisher Full Text

Russ JC, Neal FB: The Image Processing Handbook, Seventh Edition. CRC

Press, Boca Raton FL. 2015; 957-1101.

Publisher Full Text

Sage D, Donati L, Soulez F, et al: DeconvolutionLab2: An open-source software for deconvolution microscopy. Methods. 2017; 115: 28-41.

PubMed Abstract | Publisher Full Text

van Kempen GM, van Vliet LJ, Verveer PJ, et al:: A quantitative comparison of image restoration methods for confocal microscopy. J Microsc. 1997; 185(3): 354-365.

Publisher Full Text 


\section{Open Peer Review}

\section{Current Peer Review Status:}

\section{Version 2}

Reviewer Report 08 August 2017

https://doi.org/10.5256/f1000research.13353.r24822

(C) 2017 Nakano A et al. This is an open access peer review report distributed under the terms of the Creative Commons Attribution License, which permits unrestricted use, distribution, and reproduction in any medium, provided the original work is properly cited.
Akihiko Nakano
RIKEN Center for Advanced Photonics, Wako, Japan
Daisuke Miyashiro
RIKEN Center for Advanced Photonics, Wako, Japan
It was a kind of surprising outcome, but anyway it was good the SVI adequately addressed the problems. We approve that the authors' revisions are reasonable.
Competing Interests: No competing interests were disclosed.
We confirm that we have read this submission and believe that we have an appropriate level of expertise to confirm that it is of an acceptable scientific standard.

\section{Version 1}

Reviewer Report 26 June 2017

\section{https://doi.org/10.5256/f1000research.12718.r23779}

(C) 2017 Denic V et al. This is an open access peer review report distributed under the terms of the Creative Commons Attribution License, which permits unrestricted use, distribution, and reproduction in any medium, provided the original work is properly cited.

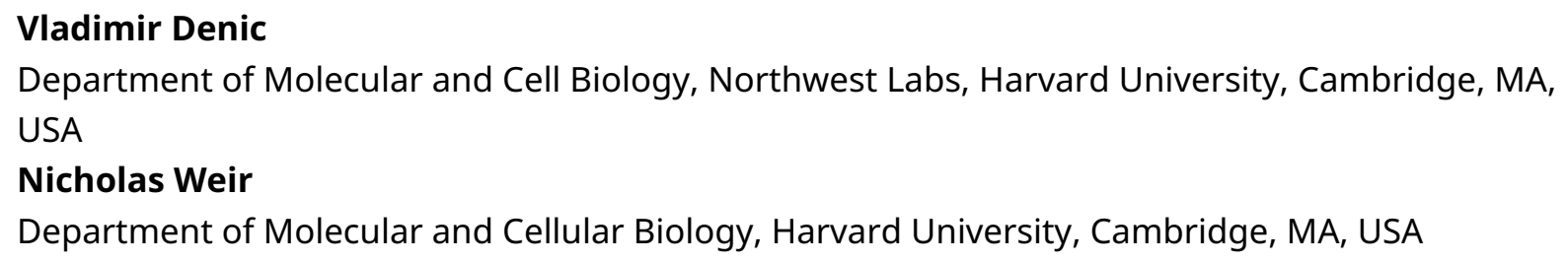


In this manuscript, Day et al. provide a protocol for preventing signal loss resulting from deconvolution of low-intensity fluorescence microscopy images by the Huygens deconvolution software. The authors convincingly demonstrate that image pre-processing using a Gaussian filter prevents loss of low-intensity fluorescent objects in the deconvolved image. Their method is welldescribed except for the omission of a few details (see below). Their experimental validation demonstrates the power of the method but might be further strengthened by small additional experiments that confirm a one-to-one correspondence between low-intensity structures derived by their image processing and the same structures derived by detection of higher-intensity signals.

Description of methods:

The authors clearly describe the rationale for their method and how it fits with pre-existing deconvolution technologies. The flow of their analysis pipeline is generally well-described, though some important details are left out:

1. How is the standard deviation (sigma) of the Gaussian filter determined? Is it empirically optimized to produce minimal loss of signal? After optimization for one image, can the same sigma be reliably applied to other images from the same dataset with consistent results?

2. The authors should address possible artifacts that may arise from spreading fluorescence detected by an SPC to adjacent pixels. Is it appropriate to artificially spread photons from single pixels to adjacent regions that may not contain excited fluorophores? How does this relate point-spread fluorescence of multiple photons to adjacent regions in high-intensity images?

Figures 3-4 contain all essential information for intepretation of the figure and do not require additional work. Minor adjustments are recommented for Figures 1 and 2, and a small additional experiment is recommended for Figure 1:

Figure 1:

Because of mobility of imaged structures within the cell, it is difficult to fully ascertain whether blurring and deconvolution of low-intensity fluorescence results in the appearance of biologically relevant structures or artificial creation of structures from background noise. This issue could in principle be resolved by: 1. repeating the experiment in Figure 1 with fixed cells in which the structures are immobile, allowing more direct comparison between visualized structures with 8 scans vs. 1 scan; or (to avoid potential cell fixation artifacts introduced by the above approach) 2 . a high-abundance protein that colocalizes with the dim structures could be fluorescently labeled in a second channel and co-visualized (alternatively, a second copy of the same protein could be fused to a bright fluor). This would help validate the expection that the blurred, deconvolved weak signal co-localizes with a stronger marker for the same structure.

Blurred, pre-deconvolution intermediate images should be shown for both the highintensity (8 scans) and low-intensity (1 scan) images.

Figure 2:

Blurred, pre-deconvolution intermediate images should be shown as indicated for Figure 1.

Is the rationale for developing the new method (or application) clearly explained? 
Yes

Is the description of the method technically sound?

Partly

Are sufficient details provided to allow replication of the method development and its use by others?

Partly

If any results are presented, are all the source data underlying the results available to ensure full reproducibility?

Yes

Are the conclusions about the method and its performance adequately supported by the findings presented in the article?

Yes

Competing Interests: No competing interests were disclosed.

We confirm that we have read this submission and believe that we have an appropriate level of expertise to confirm that it is of an acceptable scientific standard.

Author Response 02 Aug 2017

Benjamin Glick, University of Chicago, Chicago, USA

Thanks to the referees for their thoughtful feedback. Please also see the Notes to Readers for an explanation of how we approached this revision.

1) The radius (sigma) value for the Gaussian blur was optimized empirically. We found that a radius of $0.75-1.00$ pixels worked well for a variety of real and simulated confocal images. This point has been clarified in the text.

2) The pixel size in our images was $80 \mathrm{~nm}$, which is below the resolution limit of the confocal system $(\sim 200 \mathrm{~nm})$. With stronger signals, the photons from a fluorescent point source would be spread over multiple pixels. Therefore, our Gaussian blur prefilter generates patterns akin to those seen with well-sampled images.

3) We find that the best way to determine if a structure is biologically relevant is to ask whether it can be tracked between successive frames in a video. By this criterion, the videos associated with the paper confirm that most of the structures seen in the blurred and deconvolved images are indeed biologically relevant.

4) The blurred raw images are actually not very informative, and an extra panel would disrupt the 1:1 correspondence between the figures and the videos. Therefore, unless the referee feels strongly that the blurred images are essential, we would prefer to omit them. 
Competing Interests: None.

Reviewer Report 19 June 2017

https://doi.org/10.5256/f1000research.12718.r23574

(C) 2017 Nakano A et al. This is an open access peer review report distributed under the terms of the Creative Commons Attribution License, which permits unrestricted use, distribution, and reproduction in any medium, provided the original work is properly cited.

\section{Akihiko Nakano \\ RIKEN Center for Advanced Photonics, Wako, Japan \\ Daisuke Miyashiro \\ RIKEN Center for Advanced Photonics, Wako, Japan}

This paper describes a tip on using the commercial software Huygens for fluorescent image processing. The authors show that a Gaussian prefilter is useful for preserving very weak signals when the data are deconvolved by Huygens, whose algorithm cannot adequately deal with such images. From a strictly scientific point of view, we think that options of image processing including the use of filters should be discussed for general algorithms not on particular commercial software packages, because they often contain hidden algorithms. Indeed, Huygens's algorithms are not completely open. However, for most of the cell biologists who are not professional in mathematics, the use of a commercial software package for data processing is common and a tip on obtaining reasonably good-looking data may be helpful for Huygens users.

We have the following comments.

1. The deconvolution method needs be defined more clearly. Making arguments on algorithms with a black box is problematic. Can the details of maximum likelihood algorithm be disclosed, for example, by referring to a document about the method employed by Huygens? How the parameter setting was determined should also be explained.

2. To test the effects of Gaussian blurring on deconvolution of weak signals, the authors performed simulations with a generated set of fluorescent points and PSF. We agree the signal to noise ratio is very important here. In reality, the essential difference between very small numbers of measured photons and background noise must be carefully assessed. Can the authors confirm whether similar conditions are realized on actual conditions under a microscope?

3. The consideration on the unfavorable influence of the Gaussian prefilter is insufficient. The authors suggest using the filter routinely because it gives only a minor blurring effect on strong signals. This is too qualitative. Limitations of the range the method permits should be clearly stated. It will also depend on the purpose of measurements, whether quantitative numerical analysis is required with precision such as particle tracking and edge detection, 
or qualitative analysis is sufficient such as description of organelle localization dynamics.

Others are minor comments.

1. ER-decon depends on a totally different methodology and its comparison is not relevant in this paper.

2. Although the Gaussian blur prefilter has a good characteristic, the rounding error may still cause an unexpected influence on the deconvolution algorithm when the Gaussian radius is not large enough compared to the voxel size. The authors must be aware of this and may want to mention it.

Is the rationale for developing the new method (or application) clearly explained? Yes

Is the description of the method technically sound?

Partly

Are sufficient details provided to allow replication of the method development and its use by others?

Partly

If any results are presented, are all the source data underlying the results available to ensure full reproducibility?

Yes

Are the conclusions about the method and its performance adequately supported by the findings presented in the article?

Yes

Competing Interests: No competing interests were disclosed.

Reviewer Expertise: Membrane traffic, live cell imaging

We confirm that we have read this submission and believe that we have an appropriate level of expertise to confirm that it is of an acceptable scientific standard, however we have significant reservations, as outlined above.

Author Response 02 Aug 2017

Benjamin Glick, University of Chicago, Chicago, USA

Thanks to the referees for their thoughtful feedback. Please also see the Notes to Readers for an explanation of how we approached this revision.

1) The SVI website provides considerable information about their deconvolution algorithms, but Huygens is necessarily a black box to some degree. Most of the deconvolution 
parameter settings that we used were either automatic or determined by the properties of the imaging system. The SNR value is crucial, and was chosen empirically for each data set as described in the Methods.

2) The simulated data were chosen to give results similar to those we obtain from imaging yeast organelles by confocal microscopy. Noise levels in Figure 3B are actually higher than the levels we typically observe.

3) Apart from minor blurring of the final deconvolved structures, we have not noticed ill effects of a Gaussian blur prefilter under any imaging conditions. We have endeavored to emphasize this point in the text. As documented in the paper, our method preserves quantitative information about fluorescence intensities.

4) ER-Decon uses a different algorithm, but like Huygens with a Gaussian blur prefilter, it serves the purpose of deconvolving very weak fluorescence signals. The ER-Decon paper was the first to address this topic in depth and is therefore relevant for our discussion.

5) Empirically, we find that a radius of $0.75-1.00$ pixel in the Gaussian blur prefilter yields good results. This point is now emphasized more clearly in the text. Note that by using 16bit data, we avoid the rounding errors that might occur when working with smaller integer values

Competing Interests: None.

\section{Comments on this article}

\section{Version 1}

Author Response 22 Jun 2017

Benjamin Glick, University of Chicago, Chicago, USA

Thanks for the comments. In response:

1. ER-Decon is described in the Arigovindan et al. paper. That work was interesting and stimulating because it laid out the problems associated with deconvolving images that have a very low SNR. The paper includes this statement:

"Note that lambda and epsilon are the only data-dependent user-adjustable parameters, with lambda affecting smoothness and epsilon most affecting restoration of weak intensities. Ideally, both of these parameters should be optimized for each class of problem."

We found it challenging to assign a physical meaning to those parameters. More generally, the ERDecon software interface has not been optimized for usability, and it did not provide an obvious 
route for deconvolving multi-channel 4D confocal data sets.

2. The simulation involved an idealized microscopy setup in which a fluorescent object is immediately adjacent to the coverslip. The Born-Wolf model is appropriate for this configuration. For more details, please see here:

http://bigwww.epfl.ch/publications/kirshner1103.pdf

3. As described in Supplementary Figure 2, we found that a radius of 0.75 confers the full benefit of the Gaussian blur without substantially blurring the final deconvolved objects. This value for the radius was chosen empirically. A value of 0.50 was too low to obtain the full benefit. For some of the experiments, we used a radius of 1.00 with good results, so the effect is not highly sensitive to the radius value within a certain range.

We will incorporate your suggestions in a revised version.

Competing Interests: No competing interests were disclosed.

\section{Reader Comment 13 Jun 2017}

Jizhou Li, Chinese University of Hong Kong, Hong Kong

This work presents a simple but effective approach, Gaussian blur prefiltering, to improve the performance of deconvolution microscopy in very weak confocal signals.

The presentation of this paper is very good, and both the simulation and real results illustrate well the importance of this finding. The authors also provide a useful and interesting discussion.

However, there are some places are not very clear. I believe this paper could be even improved by modest changes. Specifically,

1. In page 2, the authors stated that "ER-Decon has incompletely defined parameters, and it proved to be challenging to use". It will be better if more details could be given.

2. In page 2, the authors chose the Born-Wolf model for the PSF simulation. Even though here the PSF model is not very important, it will be nice if the authors can explain a bit why this model.

3. I believe the radius of the Gaussian kernel plays an important role in the final deconvolution results (also indicated in Supplementary Figure 2). I am wondering whether the authors have a good way for choosing this parameter. It will make this finding more general to other cases.

Competing Interests: No competing interests were disclosed. 
The benefits of publishing with F1000Research:

- Your article is published within days, with no editorial bias

- You can publish traditional articles, null/negative results, case reports, data notes and more

- The peer review process is transparent and collaborative

- Your article is indexed in PubMed after passing peer review

- Dedicated customer support at every stage

For pre-submission enquiries, contact research@f1000.com 